\title{
CHEMICAL COMPOSITION OF THE ESSENTIAL OIL OF THE CROTON TETRADENIUS (EUPHORBIACEAE) AERIAL PART AND BIOACTIVITY ON AEDES AEGYPTI (DIPTERA: CULICIDAE) IN RELATION TO DIFFERENT COLLECTION PERIODS
}

\author{
ANJOS, Quirlian Queite Araújo'; SILVA, Sandra Lúcia da Cunha'; SILVA, Débora Cardoso ${ }^{1 *}$; \\ GUALBERTO, Simone Andrade ${ }^{1}$; SANTOS, Frances Regiane ${ }^{2}$; CARVALHO, Mário \\ Geraldo ${ }^{2}$; SOUSA, Daniel Lobo ${ }^{1}$
}

\author{
${ }^{1}$ Laboratório de Pesquisa de Inseticidas Naturais/Núcleo de Pesquisa em Química Aplicada/Universidade \\ Estadual do Sudoeste da Bahia, Praça Primavera, 40, Bairro Primavera, CEP 45700-000, Itapetinga - BA, \\ Brasil. (Fone: +5577 3261-8468) \\ ${ }^{2}$ Departamento de Química/ICE, Universidade Federal Rural do Rio de Janeiro/UFRRJ, Br. 465, Km 7 \\ Seropédica, CEP 23890-000, Rio de Janeiro - RJ, Brasil. \\ *Autor correspondente \\ E-mail: dcardoso_rj@hotmail.com
}

Received 08 March 2018; received in revised form 04 April 2018; accepted 04 April 2018

\section{RESUMO}

Pesquisas demonstram a presença de uma variedade de substâncias químicas nos óleos essenciais de espécies pertencentes ao gênero Croton e o seu potencial inseticida. O objetivo dessa pesquisa foi avaliar a atividade larvicida do óleo essencial obtido da parte aérea de Croton tetradenius sobre o Aedes aegypti, bem como o seu rendimento e a composição química, em relação a diferentes períodos de coleta do material botânico. Foram avaliadas oito concentrações do óleo essencial, com quatro repetições por tratamento, utilizando 30 larvas por repetição, totalizando 120 larvas por tratamento. A análise da composição química foi realizada por meio da Cromatografia Gasosa acoplada a Espectometria de Massa. O rendimento do óleo essencial na extração realizada em fevereiro foi menor. $O$ óleo essencial mais tóxico para as larvas do $A$. aegypti foi o extraído no mês de agosto. A análise da composição química revelou a presença de 60, 48 e 62 compostos nos óleos essenciais, para os meses de fevereiro, maio e agosto, respectivamente. $O$ óleo essencial obtido da parte aérea de $C$. tetradenius se mostrou promissor para ser usado em programas de controle integrado do $A$. aegypti, contudo, com vistas a maximizar esse efeito tóxico, deve-se levar em consideração o período da coleta.

Palavras-chave: Parasitologia, inseticidas botânicos, Caatinga

\section{ABSTRACT}

Research has demonstrated the presence of a variety of chemical substances in the essential oils of species belonging to the Croton genus and its potential insecticide. The objective of this research was to evaluate the larvicidal activity of the essential oil obtained from the aerial part of Croton tetradenius on Aedes aegypti, as well as its yield and chemical composition, in relation to different periods of collection of the botanical material. Eight essential oil concentrations were evaluated, using 4 replicates per treatment, using 30 larvae per replicate, totaling 120 larvae per treatment. The chemical composition analysis was carried out using Gas Chromatography coupled to Mass Spectrometry. The yield of the essential oil in the extraction carried out in February was lower. The most toxic essential oil for $A$. aegypti larvae was extracted in August. The analysis of the chemical composition revealed the presence of 60,48 and 62 compounds in the essential oils for the months of February, May and August, respectively. The essential oil obtained from the aerial part of $C$. 
tetradenius has shown to be promising for use in $A$. aegypti integrated control programs, however, in order to maximize this toxic effect, the collection period should be taken into account.

Key words: Parasitology, botanical insecticides, Caatinga

\section{INTRODUÇÃO}

Responsável por transmitir doenças que acometem, atualmente, grande parte da população mundial, o Aedes aegypti Linnaeus, 1762 (Diptera: Culicidae), é conhecido mundialmente por ser vetor em potencial dos vírus responsáveis pela dengue, febre amarela urbana, chikungunya e zika, sendo também mais recentemente associado à síndrome GuillainBarré e o aparecimento de microcefalia em recém-nascidos (TAPPE et al. 2013; VALLE et al. 2016, CIOTA et al. 2017).

Dentre essas arboviroses é importante ressaltar três: a dengue (I-IV), que acomete por ano cerca de 50 a 100 milhões de pessoas, o zika vírus, devido sua rápida expansão geográfica e as consequências ocasionadas pela mesma, inclusive no Brasil, no ano de 2016 (WHO, 2012; WHO, 2016; VALLE et al. 2016), e o vírus da febre amarela, cujo alerta epidemiológico para as Américas foi emitido pela Organização das Nações Unidas em 09 de janeiro de 2017 (WHO, 2017a).

$O$ alto índice de infestação do $A$. aegypti pode ser justificado pelas condições climáticas presentes em países de clima tropical e subtropical, e pela adaptação do mesmo ao meio urbano devido ao crescimento desordenado das cidades, aliado a diversos fatores, inclusive à falta de saneamento básico (HEMME et al. 2010; WHO, 2012; WHO, 2017b).

A estratégia de controle do $A$. aegypti mais amplamente utilizada é através do uso de inseticidas químicos, o que tem favorecido o aparecimento de populações de insetos resistentes a esses inseticidas, por conta do uso continuado desses produtos (HORTA et al. 2011). Além disso, os inseticidas químicos podem acarretar um impacto negativo no meio ambiente, interferindo no ciclo de vida de outros organismos, já que alguns desses compostos sintéticos não são seletivos (BRAGA e VALLE, 2007; GARCEZ et al. 2013).

Diante de toda a problemática que envolve 0 uso de adulticidas e larvicidas químicos sintéticos, estudos vêm sendo realizados no intuito de descobrir novos produtos, principalmente os de origem vegetal, que sejam mais seletivos, dificultem 0 aparecimento de populações de insetos resistentes e que sejam economicamente viáveis (GARCEZ et al. 2013; CRUZ et al. 2017).

As pesquisas voltadas para a utilização de produtos botânicos como inseticida baseiamse no potencial dessas espécies para produzir substancias capazes de auxiliar na manutenção destas no meio, através da proteção contra insetos, microorganismos patogênicos e herbívoros, auxiliar na atração de polinizadores e, ainda, resistir às mudanças microclimáticas (GARCÍA e CARRIL, 2011; GARCEZ et al. 2013; VASCONCELOS et al. 2017).

Dentre os produtos inseticidas naturais oriundos de famílias e gêneros botânicos utilizados sobre o $A$. aegypti, destaca-se os estudos desenvolvidos com o gênero Croton (Euphorbiaceae). Esse gênero é detentor de diversos compostos secundários, principalmente encontrados nos óleos essenciais, como relatado por Salatino et al (2007) em um estudo realizado com espécies deste gênero $e$, mais recentemente, na avaliação do potencial inseticida dessas espécies vegetais, sobre o $A$. aegypti, em estudos utilizando as espécies Croton zehntneri (Pax et Hoffm, 1923), Croton sonderianus (Muell, 1866), Croton nepetaefolius (Baill, 1891), Croton argyrophylloides (Muell, 1866) (LIMA et al. 2013), Croton linearifolius (Mull. Arg, 1873) (CUNHA et al. 2014), Croton rhamnifolioides (Pax et Hoffm, 1923) (SANTOS et al. 2014), Croton tetradenius (Baillon, 1864) (CARVALHO et al. 2016), Croton jacobinensis (Baill, 1864) (PINTO et al. 2016) e Croton argyrophyllus (Kunth, 1817) (CRUZ et al. 2017).

Dentre esses estudos, encontra-se o desenvolvido por Carvalho et al (2016), que avaliaram a atividade larvicida do óleo essencial obtido das folhas de $C$. tetradenius sobre o $A$. aegypti, a composição química desse óleo e a sua toxicidade sobre Mus musculus, demonstrando o seu potencial larvicida e uma elevada margem de segurança para o seu uso como larvicida. 
Esse estudo teve por objetivo avaliar o potencial larvicida sobre o $A$. aegypti do óleo essencial obtido das partes aéreas de $C$. tetradenius, bem como $o$ rendimento $\mathrm{e}$ a composição química do óleo essencial, em relação a diferentes períodos de coleta.

\section{MATERIAIS E MÉTODOS}

\section{Material botânico}

A parte aérea de $C$. tetradenius (folhas, flores e frutos) foi coletada na Floresta Nacional Contendas do Sincorá, localizada no município de Contendas do Sincorá, Bahia, Brasil (coordenadas geográficas S 135'02.8" W $41^{\circ} 07^{\prime} 22.0$ ")(Figura 1). A coleta foi realizada no período matutino, nos meses de Fevereiro, Maio e Agosto de 2016. A espécie foi identificada e a exsicata foi depositada no herbário da Universidade Estadual do Sudoeste da Bahia, sob o registro HUESB 3521.

Os registros médios da precipitação pluviométrica, temperatura e umidade, dos períodos de dezembro/2015 a fevereiro/ 2016, março a maio/2016 e junho a agosto/2016, foram obtidos na estação meteorológica de Ituaçu/Bahia (OMM: 83292), do Instituto Nacional de Meteorologia.

\section{Extração do óleo essencial}

Após a coleta, a parte aérea de $C$. tetradenius foi acondicionada em estufa de circulação de ar a $40^{\circ} \mathrm{C}$, durante um período de 12 horas. Posteriormente, realizou-se a extração do óleo essencial por hidrodestilação, utilizandose um extrator de Clevenger modificado a uma temperatura de $100{ }^{\circ} \mathrm{C}$, a partir de $100,0 \mathrm{~g}$ da parte aérea e $1,5 \mathrm{~L}$ de água deionizada, por um período de 3 horas. Após as extrações, adicionou-se ao óleo essencial sulfato de sódio anidro $\left(\mathrm{Na}_{2} \mathrm{SO}_{4}\right)$ com intuito de remover a água residual. Posteriormente, o óleo foi armazenado em recipiente âmbar vedado, mantido em um freezer a uma temperatura $-4^{\circ} \mathrm{C} \pm 1{ }^{\circ} \mathrm{C}$, até a realização das análises químicas e dos ensaios biológicos.

Paralelamente à extração foram avaliados o rendimento do óleo essencial e o teor de umidade, os quais foram conduzidos em triplicata. Para o cálculo do rendimento, foi utilizado o método de base livre de umidade
(BLU). Para o cálculo do teor de umidade, partiuse de 10 gramas da parte aérea, acondicionado em um balão volumétrico de fundo redondo e adicionado $70 \mathrm{~mL}$ de tolueno. Os procedimentos de avaliação do teor de umidade e do rendimento do óleo essencial seguiram a metodologia proposta por Santos et al (2004).

\section{Análise cromatográfica do óleo essencial}

As análises do óleo essencial obtido em diferentes períodos de coleta foram realizadas por meio de um cromatógrafo a gás acoplado a um Espectrômetro de Massas (Shimadzu CGEM, GC-17A/QP-2010 Plus), equipado com coluna capilar Factor Four/VF-5ms (30 m X 0,25 $\mathrm{mm}$ de diâmetro interno $X 0,25 \mu \mathrm{m}$ de espessura do filme), por meio da utilização do hélio como gás de arraste a uma vazão de $1 \mathrm{~mL} \min ^{-1} \mathrm{e}$ pressão de 12 psi. $O$ forno de temperatura foi programado de $60{ }^{\circ} \mathrm{C}$ a $260{ }^{\circ} \mathrm{C}\left(3{ }^{\circ} \mathrm{C}\right.$ min $)$, depois $10{ }^{\circ} \mathrm{C} / \mathrm{min}$ até $290{ }^{\circ} \mathrm{C}$, com temperatura do injetor a $220{ }^{\circ} \mathrm{C}$, fonte de íons a $220{ }^{\circ} \mathrm{C}$ e interface a $310^{\circ} \mathrm{C}$, posteriormente, injetou-se 1 $\mu \mathrm{L}$ de solução da amostra em diclorometano a uma razão de split 1:30. A obtenção dos espectros de massas foi realizado na faixa de varredura de 40-500 u com energia de impacto de elétrons de $70 \mathrm{eV}$. As análises quantitativas foram elaboradas utilizando um cromatógrafo de fase gasosa (HP 5890 Series II) equipado com um Detector de lonização de Chamas (DIC), nas mesmas condições experimentais e de temperatura do detector de $280^{\circ} \mathrm{C}$.

A identificação das substâncias presentes no óleo essencial de $C$. tetradenius foi realizada por meio dos seus índices de retenção (IR), calculados individualmente para cada constituinte através da injeção de uma série de padrões de hidrocarbonetos lineares (C8-C20) nas mesmas condições da amostra, e comparados com os valores tabelados (Adams, 2007), além de serem comparadas com o banco de dados da biblioteca (Nist08).

\section{Avaliação larvicida}

As larvas do $A$. aegypti utilizadas nas avaliações biológicas foram oriundas de uma colônia estabelecida no Laboratório de Pesquisa de Inseticida Naturais (LAPIN), a partir de ovos da linhagem Rockfeller, cedidos pelo Laboratório de Fisiologia e Controle de Artrópodes Vetores (LAFICAVE), da Fundação Oswaldo Cruz 
(FIOCRUZ) (Figura 2).

O preparo das concentrações foi realizado a partir da solubilização do óleo essencial, obtido em diferentes períodos de coleta (Fevereiro, Maio e Agosto), utilizando-se uma solução de Tween 80 e água deionizada a $10 \%$. Essa mesma solução também foi utilizada no grupo controle. Foram avaliadas oito concentrações $\left(0,50 \mathrm{mg} \mathrm{mL}^{-1} ; 0,25 \mathrm{mg} \mathrm{mL}^{-1}\right.$; $0,125 \mathrm{mg} \mathrm{mL}^{-1} ; 0,062 \mathrm{mg} \mathrm{mL}^{-1} ; 0,031 \mathrm{mg} \mathrm{mL}^{-1}$; $0,019 \mathrm{mg} \mathrm{mL}^{-1} ; 0,007 \mathrm{mg} \mathrm{mL}^{-1}$ e $0,003 \mathrm{mg} \mathrm{mL}^{-1}$ ), com quatro repetições por tratamento.

Utilizou-se 30 larvas do $A$. aegypti por repetição, entre o terceiro e o quarto estádio. As larvas foram imersas em $29 \mathrm{~mL}$ de água deionizada, na qual foi adicionada $1 \mathrm{~mL}$ das diferentes concentrações. Os horários de observações da mortalidade larval foram realizados com 2, 4, 8, 16 e 24 horas, após o início do experimento.

Os percentuais de mortalidade das larvas do $A$. aegypti em relação às diferentes concentrações, dentro de um mesmo período de coleta, foram submetidos ao teste de Tukey, ao nível de $5 \%$ de probabilidade. A comparação da mortalidade larval nos diferentes períodos de coleta foi realizada a partir da avaliação da sobreposição dos intervalos de confiança.

\section{RESULTADOS E DISCUSSÃO}

Ao avaliar a sobreposição dos intervalos de confiança, verificou-se que não houve diferença significativa entre os teores de umidade de Fevereiro (10,67\%), Maio $(11,33 \%)$ e Agosto $(11,67 \%)$. Contudo, o rendimento médio para o óleo essencial extraído no mês de fevereiro foi significativamente menor (1.98\%), quando comparados aos meses de maio $(2,99 \%)$ e agosto $(3,13 \%)$ (Tabela 1$)$.

Segundo Gobbo-Neto e Lopes (2007) e Natta et al (2008), diversos fatores podem interferir na produção do óleo essencial, sendo citados características como a idade, o estádio de desenvolvimento, as interações interespecíficas e intraespecíficas, assim como os fatores abióticos, tais como: condições edáficas, temperatura, umidade, luminosidade e índice pluviométrico.

Dentre esses fatores, as condições edafoclimáticas podem ter interferido de maneira significativa, haja vista que a espécie alvo desse estudo foi coletada em uma região da Caatinga, cujo período mais seco pode agravar as condições edáficas, modificando a fisiologia da planta e interferindo na produção dos metabólitos secundários, tanto em nível quantitativo quanto qualitativo.

Ao verificar os registros médios da precipitação pluviométrica, temperatura e umidade da região onde foi realizada a coleta da parte aérea de $C$. tetradenius, o período compreendido entre dezembro de 2015 a fevereiro de 2016 apresentou uma maior precipitação pluviométrica média $(127,43 \%)$, comparada aos períodos de Março a Maio de 2016 (2,80\%) e Junho a Agosto de 2016 (6,67\%), sendo que entre esses dois últimos períodos, não houve diferença significativa. Com relação às temperaturas e umidades, não foi observado uma diferença significativa entre os três períodos avaliados (Tabela 2).

Um estudo conduzido por Carvalho et al (2016), revelou que o rendimento médio do óleo essencial obtido das folhas de $C$. tetradenius, cuja coleta foi realizada em maio de 2014 , foi maior $(2,73 \%)$ que o verificado nesse estudo $(1,98 \%)$, na coleta realizada em fevereiro de 2016 (Tabela 1). Vale destacar que os referidos autores realizaram o experimento somente com as folhas, diferentemente desse estudo, que foi realizado com a parte aérea (folhas, flores e frutos), o que, provavelmente, pode ter interferido no rendimento do óleo essencial.

Por outro lado, a precipitação pluviométrica média, obtida no Instituto Nacional de Meteorologia, para o trimestre de dezembro/2013 a fevereiro/2014, que correspondeu a coleta realizada por Carvalho et al (2016), foi inferior $(54,23 \mathrm{~mm})$ ao trimestre de dezembro/2015 a fevereiro/2016, período em que foi realizada a coleta nesse estudo $(127,43 \mathrm{~mm})$. No caso da coleta realizada por Carvalho et al (2016), a maior precipitação ocorreu no mês de dezembro $(150,4 \mathrm{~mm})$, e na coleta realizada nesse estudo foi no mês de janeiro (366.2 $\mathrm{mm}$ ). Esses fatores podem, também, ter interferido na produção dos metabólitos secundários e, consequentemente, no rendimento do óleo essencial.

De acordo com Morais (2009) o estresse hídrico pode afetar diversos fatores fisiológicos 
no vegetal, como o crescimento e a expansão foliar, podendo gerar alterações no metabolismo secundário. Outro aspecto que pode alterar o rendimento do óleo essencial, destacado pela autora, são as chuvas intensas e constantes que podem carrear substâncias hidrossolúveis presentes nas folhas e flores.

Solos encharcados também podem ficar ausentes de oxigênio o que faz com que as raízes realizem a respiração anaeróbia, que tem por consequência a diminuição do crescimento radicular e menos absorção de água e nutrientes, de acordo com Pes e Arenhardt (2015), o que pode também afetar o metabolismo das plantas $\mathrm{e}$, consequentemente, $\mathrm{o}$ rendimento do óleo essencial.

Cruz et al (2017) avaliaram o rendimento do óleo essencial obtido das folhas de $C$. argyrophyllus, coletado no mês de maio de 2014 , e obtiveram um valor de $0,48 \%$. Outros trabalhos realizados com folhas de espécies de Croton também apresentaram rendimento inferior ao obtido nesse estudo, como o realizado por Dória et al (2010), cujo rendimento do óleo essencial de Croton heliotropiifolius (Kunth, 1817) foi $0,2 \%$ e o realizado por Santos et al (2014), que avaliando o rendimento do óleo essencial de $C$. rhamnifolioides obtiveram um percentual de $0,08 \%$.

O óleo essencial de $C$. tetradenius, cuja parte aérea foi coletada no mês de fevereiro, não ocasionou mortalidade larval significativa em nenhuma das concentrações no período de 2 horas de exposição. Somente a partir de 4 horas de exposição das larvas, foi possível verificar uma mortalidade de $63,33 \%$ na concentração de $0,50 \mathrm{mg} \mathrm{mL}^{-1}$, sendo significativamente mais tóxica para as larvas quando comparado às demais concentrações. Essa mesma concentração manteve-se significativamente mais tóxica $(p<0,05)$, ocasionando $97,50 \%$ de mortalidade larval, com 8 horas de exposição e $100,00 \%$ a partir de 16 horas de exposição. A segunda concentração mais efetiva $\left(0,25 \mathrm{mg} \mathrm{mL}^{-}\right.$ 1) ocasionou com 24 horas de exposição uma mortalidade de $46,66 \%$. As demais concentrações não se mostraram tóxicas para as larvas do $A$. aegypti (Quadro 1).

A concentração de $0,50 \mathrm{mg} \mathrm{mL}^{-1}$ do óleo essencial cujas partes aéreas de $C$. tetradenius foram coletadas no mês de maio, também foi significativamente mais efetiva para as larvas do
A. aegypti, comparado às demais concentrações $(p<0,05)$. Com 8 horas de exposição ocasionou $100,00 \%$ de mortalidade das larvas. A concentração de $0,25 \mathrm{mg} \mathrm{mL}^{-1}$, com 24 horas de exposição das larvas, ocasionou $8,33 \%$ de mortalidade. Não houve mortalidade larval nas demais concentrações e no grupo controle (Tabela 3).

Com relação à avaliação do óleo essencial obtido a partir da coleta das partes aéreas de $C$. tetradenius no mês de agosto, com 4 horas de exposição à concentração de $0,50 \mathrm{mg}$ $\mathrm{mL}^{-1}$ ocasionou $100,00 \%$ de mortalidade larval, sendo significativamente mais tóxica para o $A$. aegypti $(\mathrm{p}<0,05)$. A concentração de $0,25 \mathrm{mg} \mathrm{mL}^{-}$ ${ }^{1}$ foi a segunda significativamente mais efetiva, ocasionando com 16 horas de exposição 55,83\% de mortalidade larval e com 24 horas $80,00 \%$ de mortalidade $(p<0,05)$. Nas demais concentrações não houve mortalidade larval (Tabela 3 ).

Ao comparar o percentual de mortalidade larval entre os três períodos de coleta das partes aéreas (fevereiro, maio e agosto), a partir da análise da sobreposição dos intervalos de confiança, observou-se que, com 2 e 4 horas de exposição das larvas, a concentração de $0,50 \mathrm{mg} \mathrm{mL}^{-1}$ oriunda da coleta realizada no mês de agosto, foi mais tóxica para as larvas do $A$. aegypti, comparada aos meses de fevereiro e maio. A partir de 8 horas de exposição não houve diferença significativa na mortalidade larval em relação aos diferentes períodos de coleta (Tabela 3).

Contudo, na concentração de $0,25 \mathrm{mg}$ $\mathrm{mL}^{-1}$, as coletas realizadas nos meses de fevereiro e agosto foram significativamente mais efetivas comparada a coleta realizada no mês de maio, nos períodos de exposição larval de 8 e 16 horas. Com 24 horas de exposição das larvas, a coleta realizada no mês de agosto foi significativamente mais tóxica para as larvas do A. aegypti (Tabela 3$)$.

A análise da composição química do óleo essencial obtido das partes aéreas de $C$. tetradenius coletada no mês de fevereiro, indicou a presença de 60 compostos, tendo sido identificados 37 compostos, correspondendo a 61,66\%, classificados como: monoterpenos hidrocarbonados $(37,83 \%)$, monoterpenos oxigenados $(35,13 \%), \quad$ sesquiterpenos hidrocarbonados $(16,21 \%)$ e sesquiterpenos oxigenados $(10,81 \%)$ (Tabela 4). Dentre as 
substâncias identificadas, foram consideradas como majoritárias as que apresentaram percentuais acima de 1\%: Cânfora (18,18\%), YTerpineol (9,76\%), a-Terpineno (6,99\%), pCimeno (5,52\%), $y$-Terpineno (4,72\%), Mirceno (4,38\%), Limoneno (3,45\%), $\alpha$-Felandreno $(3,05 \%)$, a-Pineno $(3,21 \%)$, Canfeno $(2,72 \%)$, Terpineno-4-ol (2,57\%), Isoborneol (2,34\%), aTerpinil acetato $(2,15 \%)$, $\alpha$-Copaeno $(2,04 \%)$, $\alpha$ Humuleno (1,66\%), Biciclogermacreno $(1,32 \%)$, Sabineno $(1,29 \%)$, Tricicleno $(1,25 \%)$, $\alpha$-Tujeno $(1,25 \%)$, Espatulenol (1,17\%), @-2-Careno $(1,11 \%)$, conforme apresentado na Tabela 5.

A análise química do óleo essencial extraído no mês de maio indicou a presença de 48 compostos. Desses, 30 foram identificados, correspondendo a $62,50 \%$, sendo $40,00 \%$ de monoterpenos hidrocarbonados, $26,66 \%$ de monoterpenos oxigenados, $20,00 \%$ de sesquiterpenos hidrocarbonados e 13,33\% de sesquiterpenos oxigenados (Tabela 4). Como componentes majoritários têm-se: Cânfora $(30,95 \%), \quad y$-Terpineol $(12,70 \%), \quad p$-Cimeno $(6,95 \%)$, o Isoborneol $(4,54 \%)$, Terpineno-4-ol $(3,66 \%), y$ - Terpineno $(3,44 \%), \alpha$-Terpinil acetate (3,07\%), a-Copaeno (2,98\%), Limoneno $(1,96 \%)$, Mirceno $(1,80 \%)$, $\alpha$-Terpineno $(1,78 \%), \alpha-$ Humuleno (1,74\%), $\beta$-Cariofileno $(1,16 \%)$ e Mirtenil acetato $(1,01)$ (Tabela 5).

A análise da composição química do óleo essencial extraído no mês de agosto indicou a presença de 62 compostos, tendo sido identificados 36 compostos $(58,06 \%)$, sendo: $38,88 \%$ de monoterpenos hidrocarbonados, $36,11 \%$ de monoterpenos oxigenados, $16,67 \%$ de sesquiterpenos hidrocarbonados e $8,33 \%$ de sesquiterpenos oxigenados (Tabela 4). Como componentes majoritários foram encontrados: Cânfora $(23,91 \%), \quad y$-Terpineol $(16,00 \%), \quad p$ Cimeno (7,85\%), a-Pineno (3,88\%), Limoneno $(3,51 \%)$, Canfeno $(3,50 \%)$, $y$-Terpineno $(3,15 \%)$, Mirceno $(3,05 \%)$, Isoborneol $(2,34 \%)$, Terpineno4-ol (2,31\%), a-Terpineno (2,24\%), a-Copaeno $(1,78 \%)$, Tricicleno $(1,53 \%), \alpha-T e r p i n i l ~ a c e t a t o$ $(1,48 \%)$ e $\alpha$-Felandreno $(1,37 \%)$ (Tabela 5$)$.

Dos compostos identificados que apresentaram percentuais acima de $1 \%$, o Tricicleno, $\alpha$-Pineno, Canfeno e o $\alpha$-Felandreno somente foram detectados, nesse percentual, nos óleos essenciais cuja coleta da parte aérea de $C$. tetradenius foi realizada nos meses de fevereiro e agosto. Dos compostos que apresentaram percentuais abaixo de 1\%, 1,8-
Cineol, trans-p-Mentha-2,8-dien-1-ol, cis-pMentha-2,8-dien-1-ol, Pinocarvone e $\alpha$-Terpineol também foram encontrados somente nas coletas de fevereiro e agosto (Tabela 5).

Pesquisas têm demonstrado 0 potencial inseticida e farmacológico de espécies pertencentes ao gênero Croton, especialmente no bioma Caatinga, sendo este gênero detentor de inúmeros compostos químicos, ressaltando, assim, a necessidade do desenvolvimento de programas e projetos voltados para o uso e a conservação das espécies encontradas nesse bioma. Muitas dessas espécies têm potencial para serem utilizadas em programas de controle integrado do $A$. aegypti, transmissor em potencial dos vírus da dengue, chikungunya e zika, como as espécies $C$. zehntneri, $C$. sonderianus e $C$. argyrophylloides (LIMA et al, 2013), C. tetradenius (CARVALHO et al, 2016), C. argyrophyllus (CRUZ et al, 2017) e C. nepetaefolius (SANTOS et al, 2017).

Carvalho et al (2016) analisando a composição química do óleo essencial cujas folhas de $C$. tetradenius foram coletadas no mês de maio de 2014, detectaram a presença de 26 compostos, diferentemente do encontrado no presente estudo, onde, nesse mesmo período, foram encontrados 48 compostos, tendo nesses dois estudos a Cânfora como a substância com maior percentual.

A diversidade de substância encontradas na parte aérea de $C$. tetradenius, assim como as diferenças quantitativas dos compostos, comparado ao estudo desenvolvido por Carvalho et al (2016), pode estar relacionado a diversos fatores, como a parte anatômica avaliada, a constituição genética, o estádio de desenvolvimento e as condições edafoclimáticas. O índice pluviométrico pode ter interferido nesses resultados, conforme já destacado.

Cerqueira et al (2009), ao avaliar a influencia dos fatores climáticos na composição química do óleo essencial das folhas de Myrcia salzmannii (Berg., 1857) (Myrtaceae), verificaram, através da comparação do óleo essencial obtido em diferentes períodos de coleta, uma variação na sua constituição química, inferindo que o índice pluviométrico pode influenciar na concentração de alguns compostos.

O óleo essencial obtido a partir da coleta realizada no mês de maio não se mostrou tão 
eficaz comparado aos meses de fevereiro e agosto, sendo que destes, o óleo essencial cuja parte aérea foi coletada no mês de agosto se mostrou mais tóxico para as larvas do $A$. aegypti, o que pode estar relacionado com a composição química do óleo, tanto em termos quantitativos quanto qualitativo. A presença de uma substância, seja majoritária ou não, pode intensificar ou até mesmo inibir a ação inseticida, portanto, na avaliação inseticida de uma planta, deve ser considerado o sinergismo entre os compostos, assim como o antagonismo.

Dos compostos identificados, a Cânfora foi o que apresentou o maior percentual dentre os compostos encontrados nas três coletas realizadas, sendo que o maior percentual ocorreu no óleo essencial cuja parte aérea foi coletada no mês de maio $(30,95 \%)$ e, nem por isso, esse óleo se mostrou mais tóxico para as larvas do $A$. aegypti.

Ali et al (2015a) verificaram que mesmo havendo a presença do composto Cânfora no percentual de $4,4 \%$ no óleo essencial de Salvia apiana (Jeps, 1908), este não foi tóxico para o $A$. aegypti, quando comparado aos outros óleos essenciais avaliados que tiveram atividade sobre o A. aegypti e nos quais não foi encontrada essa substância. Segundo Park et al (2011), o potencial toxicológico presente em óleos essenciais extraídos de alguns exemplares botânicos, pode ser devido ao efeito sinérgico presente em substancias encontradas em concentrações menores, o que demonstra a necessidade de se avaliar de forma mais criteriosa essas interações.

O maior percentual de $\alpha$-Pineno encontrado nos óleos essenciais cuja parte aérea foi coletada nos meses de fevereiro e agosto pode ter contribuído com o maior efeito tóxico sobre as larvas do $A$. aegypti desses óleos essenciais, comparado ao óleo essencial obtido no mês de maio. Singh et al (2006) avaliando o efeito inibitório do a-pineno sobre o crescimento radicular, observou que esse composto inibe o crescimento precoce das raízes e causa dano oxidativo no tecido radicular. Um estudo desenvolvido por Leite et al (2017) também indicou que o $\alpha$-Pineno afeta a permeabilidade da membrana celular e que esse efeito é dependente da concentração desse composto. Efeito similar pode ter ocorrido com as larvas do A. aegypti.
O 1,8-Cineol embora presente em uma concentração inferior a $1 \%$, também pode ter contribuído com a maior toxicidade dos óleos essenciais obtidos a partir das coletas realizadas nos meses de fevereiro e agosto, visto que essa substância não foi encontrada no óleo essencial extraído no mês de maio. Contudo, 1,8-Cineol foi encontrado como composto majoritário $(71,7 \%)$ por Ali et al (2015a), no óleo essencial obtido das partes aéreas de $S$. apiana, o qual não se mostrou tóxico para as larvas do $A$. aegypti e Anopheles quadrimaculatus (Say, 1824). Essa mesma substância foi encontrada nos óleos essenciais de Salvia elegans (Vahl, 1804) e Salvia officinalis (Linneaus, 1753), em concentrações bem inferiores $(3,5 \%$ e $4,4 \%$, respectivamente), e, mesmo assim, esses óleos apresentaram efeito tóxico sobre as larvas. Esses dados revelam a importância de ter um olhar sistêmico quando se pensa na formulação de um inseticida botânico, pois a avaliação de uma substância isolada pode não revelar a sua real contribuição no efeito tóxico da espécie que se está avaliando, pois uma substância pode potencializar esse efeito tóxico atuando de forma sinérgica (adição ou potenciação) ou de forma antagônica.

Os compostos a-terpineol e pinocarvone apesar de serem compostos minoritários, podem, também, estar contribuindo para uma melhor atividade larvicida dos óleos essenciais de $C$. tetradenius, obtidos das coletas realizadas em fevereiro e agosto, visto que essas substâncias não foram encontradas no óleo essencial cuja parte aérea foi coletada no mês de maio.

O composto Canfeno embora presente em todas as coletas, o menor percentual para este foi obtido no óleo essencial coletado no mês de maio, com percentual inferior a $1 \%$. Este fato também pode estar relacionado com a maior toxicidade presente nos óleos essenciais coletados em fevereiro e agosto, tendo em vista que este composto apresentou, para essas duas coletas, percentuais de $2,72 \%$ e $3,50 \%$, respectivamente, enquanto que para o mês de maio foi de $0,92 \%$.

O a-Felandreno foi encontrado como composto majoritário somente nos óleos essenciais extraídos nos meses de fevereiro e agosto, sendo que no mês de maio, esse constituinte apresentou um percentual de $0,79 \%$. Ali et al (2015b) comprovaram a eficiência deste composto sobre larvas do $A$. aegypti, quando 
comparado com outras substâncias presente no óleo essencial extraído de diferentes estruturas vegetais de Echinophora lamondiana (B.Yildiz, 1997).

Mesmo que o composto limoneno tenha sido identificado nos três períodos de extração dos óleos essenciais como majoritário, os meses de fevereiro e agosto apresentaram percentuais mais elevados dessa substância $(3,45 \%$ e $3,51 \%$, respectivamente), comparado ao mês de maio $(1,96 \%)$, podendo este, também, estar contribuindo com a maior eficiência dos óleos essenciais coletados em fevereiro e agosto. Park et al (2011), verificaram o potencial tóxico desse composto presente nos óleos essenciais de Melaleuca dissitiflora (F. Muell, 1863), Melaleuca quinquenervia (Cav.) S.T. Blake (1958), Melaleuca linariifolia (Cav.) S.T. Blake (1958) e Eucalyptus globulus (Labill, 1800), sobre larvas do A. aegypti.

Cheng et al (2009) avaliaram a atividade larvicida de 12 compostos sobre o $A$. aegypti, dentre eles o 1,8-cineol, $\alpha$-pineno, $\alpha$-terpinilo, $\alpha$ terpineol, terpinen-4-ol e $\beta$-eudesmol apresentaram uma $\mathrm{Cl}_{50}$ maior que $50,0 \mu \mathrm{g} / \mathrm{mL}$, sendo consideradas não tóxicas para as larvas. Contudo, $\alpha$-Felandreno, Limoneno, $p$-cimeno, $y^{-}$ terpineno, terpinoleno e $\alpha$-terpineno apresentaram forte atividade sobre as larvas do A. aegypti. Entre estes constituintes, 0 aterpineno apresentou maior toxicidade para as larvas do $A$. aegypti, seguido do a-Felandreno.

Vale ressaltar que, tanto em termos qualitativo quanto quantitativo, os compostos encontrados nos óleos essenciais de $C$. tetradenius, nos diferentes períodos de coleta das partes aéreas, possam ser um indicativo de uma provável atuação no efeito tóxico encontrado nas coletas de fevereiro e agosto, comparado à coleta do mês de maio, há que se considerar a interação entre os diferentes compostos, o que pode intensificar ou diminuir o efeito tóxico sobre as larvas do $A$. aegypti.

Seo et al (2012), avaliando a atividade larvicida de constituintes químicos oriundos do óleo essencial de Trachyspermum ammi (Linneaus, 1753), bem como de seus blends, sobre larvas do $A$. aegypti, verificaram que a eliminação do timol, $p$-cimeno e $y$-terpineno do blend ocasionou uma diminuição da toxicidade sobre o $A$. aegypti, indicando um provável sinergismo entre compostos.

\section{CONCLUSÕES:}

O óleo essencial extraído da parte aérea de $C$. tetradenius possui efeito tóxico sobre as larvas do $A$. aegypti. O período de coleta da parte aérea de $C$. tetradenius afeta o rendimento do óleo essencial e a sua composição química, assim como a sua toxicidade sobre as larvas do Aedes aegypti. Dessa forma, embora o óleo essencial de $C$. tetradenius tenha potencial para ser utilizado em programas de controle integrado do $A$. aegypti, diversos fatores, como os edafoclimáticos, assim como o horário da coleta e o estádio do desenvolvimento, dentre outros, devem ser levados em consideração quando se pensa na formulação e comercialização dessa espécie como inseticida botânico, com vistas a padronizar, tanto em termos quantitativos quanto qualitativos, a sua composição química, garantindo, assim, a efetividade do produto.

\section{AGRADECIMENTOS:}

À Coordenação de Aperfeiçoamento de Pessoal de Nível Superior (CAPES), à Universidade Estadual do Sudoeste da Bahia (UESB), a Fundação de Amparo a Pesquisa do Estado da Bahia (FAPESB).

\section{REFERÊNCIAS:}

1. Adams, R. P. Allured Publishing Corporation: Carol Stream, 2007, 4 ed.

2. Ali, A.; Tabanca, N.; Demirci, B.; Blythe, E. K.; Ali, Z.; Baser, K. H. C.; Khan, I. A.; J. Agric. Food Chem. 2015a, 63(2), 447-456.

3. Ali, A.; Tabanca, N.; Ozek, G.; Ozek, T.; Aytac, Z.; Bernier, U. R.; Khan, I. A.; J. Med. Entomol. 2015b, 52(1), 93-100.

4. Braga, I. A.; Valle, D.; Epidemiologia e Serviços de Saúde . 2007,16(4), 179-293.

5. Carvalho, K. S.; Silva, S. L. D. C.; Souza, I. A.; Gualberto, S. A.; Cruz, R. C. D.; Dos Santos, F. R.; Carvalho, M. G.; Parasitol. Res. 2016, 115(9), 3441-3448.

6. Cerqueira, M. D. D.; Marques, E. D. J.; Martins, D.; Roque, N. F.; Cruz, F. G.; Guedes, M. L. D. S.; Quim. Nova. 2009, 32(6), 1544-1548. 
7. Cheng, S. S.; Huang, C. G.; Chen, Y. J.; Yu, J. J.; Chen, W. J.; \& Chang, S. T.; Bioresour.Technol. 2009, 100(1), 452456.

8. Ciota, A. T.; Bialosuknia, S. M.; Ehrbar, D. J.; Kramer, L. D.; Emerging Infect. Dis. 2017, 23(5), 880.

9. Cruz, R. C. D.; Cunha, S. L. S.; Souza, I. A.; Gualberto, S. A.; Carvalho, K. S.; Santos, F. R.; Carvalho, M. G.; J. Med. Entomol. 2017, 54(4), 985-993.

10. Cunha, S. L. S.; Gualberto, S. A.; Carvalho, K. S.; Fries, D. D.; Biotemas. 2014, 27(2), 79-85.

11. Dória, G. A.; Silva, W. J.; Carvalho, G. A.; Alves, P. B.; Pharm. Biol. 2010, 48(6), 615-620.

12. Garcez, W. S.; Garcez, F. R.; Da Silva, L. M.; Sarmento, U. C.; Rev. Virtual Quim. 2013, 5(3), 363-393.

13. García, A. Á.; Carril, E. P. U.; Reduca (biología). 2011, 2(3).

14. Gobbo-Neto, L.; Lopes, N. P.; Quim. Nova. 2007, 30 (2), 374.

15. Hemme, R. R.; Thomas, C. L.; Chadee D. D.; Severson, D. W.; PLoS Neglected Trop. Dis. 2010, 4(3), 634.

16. Horta, M. A. P.; Castro, F. I.; Rosa, C. S.; Daniel, M. C.; Melo, A. L.; BioAssay. 2011, 6.

17. Leite, T. R.; Silva, M. A. P. D.; Santos, A. C. B. D.; Coutinho, H. D. M.; Duarte, A. E.; Costa, J. G. M. D.; Pharm. Biol. 2017, 55(1), 2015-2019.

18. Lima, G. P. G.; De Souza, T. M.; De Paula Freire, G.; Farias, D. F.; Cunha, A. P.; Ricardo, N. M. P. S.; Carvalho, A. F. U.; Parasitol. Res. 2013, 112(5), 1953-1958.

19. Morais, L. A. S.; Influência dos fatores abióticos na composição química dos óleos essenciais. Hortic. Bras. 2009, $27(2)$
20. Morais, S. M.; Cavalcanti, E. S.; Bertini, L. M.; Oliveira, C. L. L.; Rodrigues, J. R. B.; Cardoso, J. H. L.; J. Am. Mosq. Control Assoc. 2006, 22(1), 161-164.

21. Natta, L.; Orapin, K.; Krittika, N.; Pantip, B.; Int. Food Res. J. 2008, 15(3),1-10.

22. Park, H. M.; Kim, J.; Chang, K. S.; Kim, B. S.; Yang, Y. J.; Kim, G. H.; Park, I. K.; J. Med. Entomol. 2011, 48 (2), 405-410.

23. Pes, L. Z.; Arenhardt, M. H. Rede e-Tec, Brasil, 2015.

24. Pinto, C. C. C.; Sa De Menezes, J. E.; Siqueira, C. S. M.; Melo, D. S.; Feitosa, C. R.; Santos, H. S.; Bol. Latinoam. Caribe Plant. Med. Aromat. 2016, 15(2).

25. Salatino, A.; Salatino, M. L. F.; Negri, G. Journal Of The Brazilian Chemical Society, 2007, V. 18, N. 1, P. 11-33.

26. Santos, A. S.; Alves, S. M.; Figueiredo, F. J.; Rocha Neto, G.; EMBRAPA. 2004, 1-6.

27. Santos, G. K. N.; Dutra, K. A.; Lira, C. S.; Lima, B. N.; Napoleão, T. H.; Paiva, P. M. G.; Maranhão, C. A.; Brandão, S. S. F.; Navarro, D. M. A. F.; Molecules. 2014, 19(10),16573-16587.

28. Santos, H. S.; Bandeira, P. N.; Lemos, T. L.; Santiago, G. M. International Journal of Mosquito, 2017, p. 19-22.

29. Seo, S. M. I; Park, Hye. M. I.; Park, I. L. K.; J. Agric. Food Chem. 2012, 60(23) 5909-5914.

30. Silva, A. A. S.; Silva, A. A. S.; Morais, S. M.; Martins, C. G.; Vieira, F. M. A.; Rev. Eletronica Farm. 2016, 13(3),165-171.

31. Singh, H. P.; Batish, D. R.; Kaur, S.; Arora, K.; Kohli, R. K.; Ann. Bot. (Oxford, U. K.). 2006, 98(6), 1261-1269.

32. Tappe, D. R. J.; Gabriel, M.; Emmerich, P. G. S.; Held, G.; Smola, S.; SchmidtChanasit, J. L.; Eurosurveillance. 2013, 54(54).

33. Valle, D.; Pimenta, D. N.; Aguiar, R.; Epidemiologia e Serviços de Saúde. 
2016, 25(2),419-422.

34. Vasconcelos, E. A. F.; De Freitas Mesquita, A. K.; Citó, A. M. D. G. L.; Lopes, J. A. D.; Vita et Sanitas. 2017, $7(1), 123-134$.

35. World Health Organization. Alerta Epidemiológica Fiebre amarilla. 2017a.

36. World Health Organization. Global strategy for dengue prevention and control. 2012.

37. World Health Organization. Global vector control response. 2017b.

38. World Health Organization. Zika strategic response plan quarterly update. 2016. 


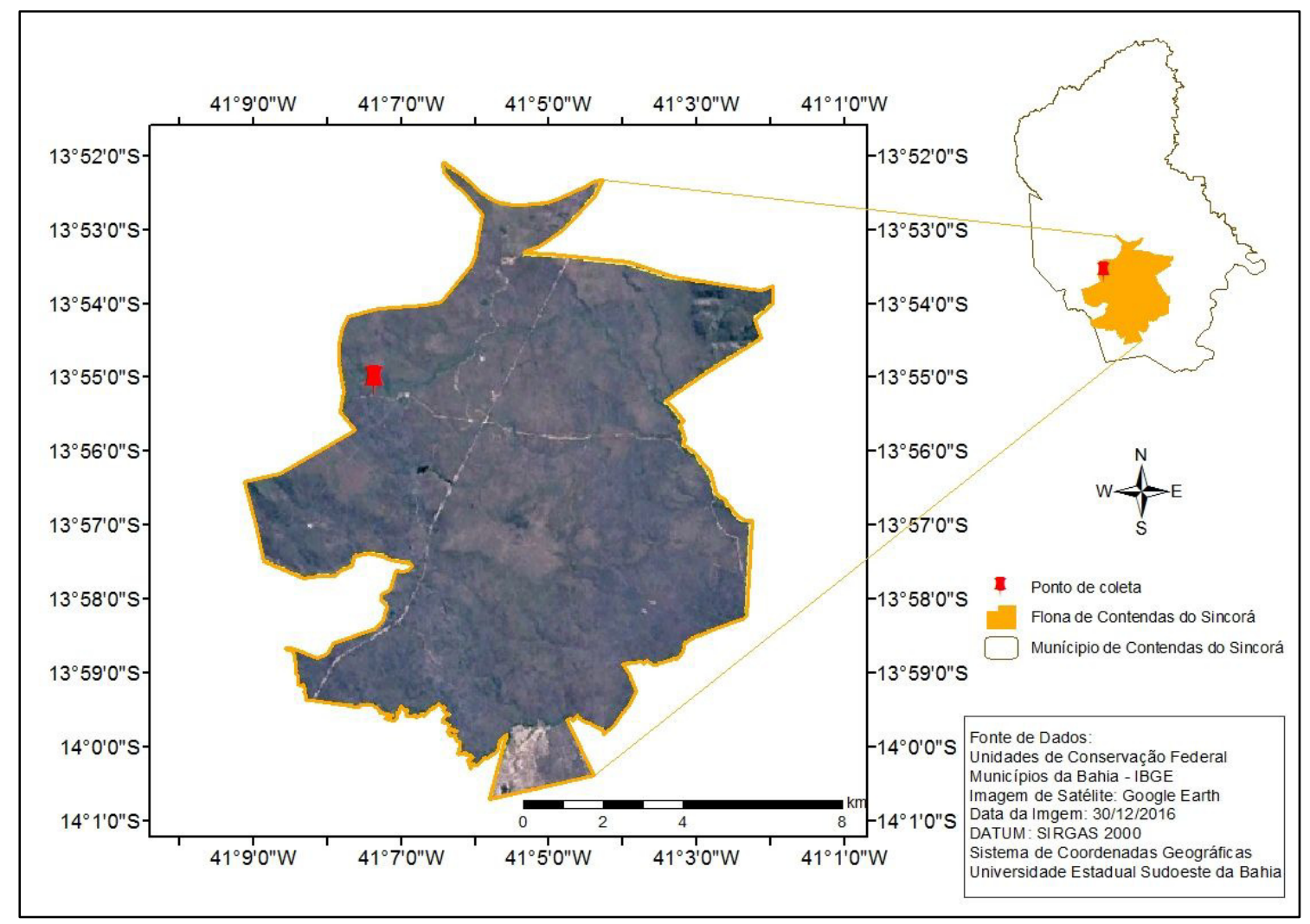

Figura 1. Local de coleta das partes aéreas de Croton tetradenius. Floresta Nacional Contendas do Sincorá, município de Contendas do Sincorá, Bahia, Brasil

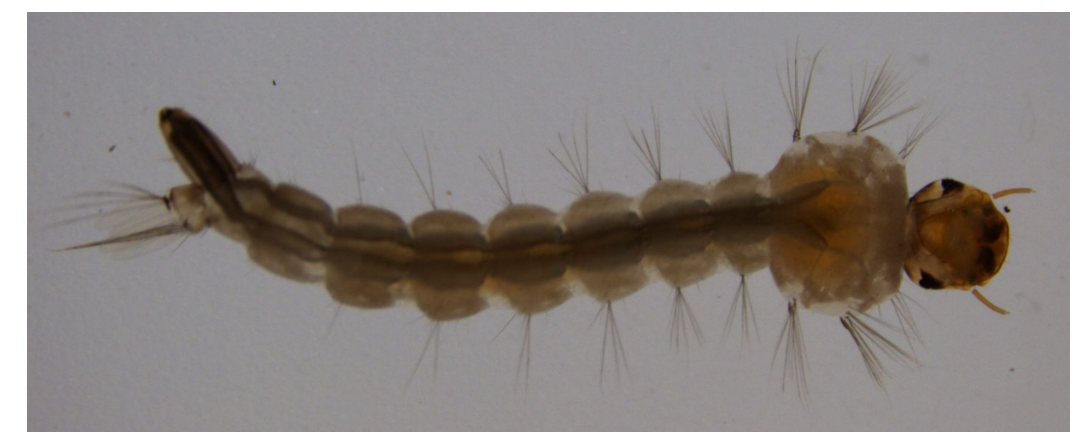

Figura 2. Larva do Aedes aegypti, linhagem Rockfeller, oriunda da colônia mantida no Laboratório de Pesquisa de Inseticidas Naturais (LAPIN), da Universidade Estadual do Sudoeste da Bahia/Campus de Itapetinga. 
Tabela 1. Teor de umidade e rendimento do óleo essencial da parte aérea de Croton tetradenius, em relação a três diferentes períodos de coletas

\begin{tabular}{ccccc}
\hline $\begin{array}{c}\text { Período } \\
\text { de coleta }\end{array}$ & $\begin{array}{c}\text { Teor de } \\
\text { Umidade } \\
\text { Médio (\%) }\end{array}$ & I.C. ${ }^{1}$ & $\begin{array}{c}\text { Rendimento } \\
\text { Médio } \\
(\%)\end{array}$ & I.C. $^{1}$ \\
\hline Fevereiro & 10,67 & $5,96-15,38$ & 1,98 & $1,90-2,06$ \\
Maio & 11,33 & $2,19-20,48$ & 2,99 & $2,50-3,49$ \\
Agosto & 11,67 & $8,40-14,93$ & 3,13 & $3,00-3,27$ \\
\hline I.C. = Intervalo de Confiança. & & &
\end{tabular}

Tabela 2. Registros médios da precipitação pluviométrica, temperatura e umidade, obtidas na estação meteorológica de Ituaçu/Bahia (OMM: 83292), do Instituto Nacional de Meteorologia

\begin{tabular}{|c|c|c|c|c|c|c|}
\hline Período & $\begin{array}{c}\text { Precipitação } \\
\text { Média } \\
\text { (mm) }\end{array}$ & I.C. ${ }^{1}$ & $\begin{array}{c}\text { Temperatura } \\
\text { Média } \\
\left({ }^{\circ} \mathrm{C}\right)\end{array}$ & I.C. ${ }^{1}$ & $\begin{array}{l}\text { Umidade } \\
\text { Média (\%) }\end{array}$ & I.C. ${ }^{1}$ \\
\hline $\begin{array}{c}\text { Dezembro } \\
\text { /2015 a } \\
\text { Fevereiro/ } \\
2016\end{array}$ & 127,43 & $\begin{array}{c}106,64 \\
- \\
361,51\end{array}$ & 26,07 & $\begin{array}{c}24,37 \\
- \\
27,78\end{array}$ & 56,24 & $\begin{array}{c}42,78 \\
- \\
69,70\end{array}$ \\
\hline $\begin{array}{c}\text { Março a } \\
\text { Maio/2016 }\end{array}$ & 2,80 & $\begin{array}{l}0,81 \\
- \\
4,79\end{array}$ & 24,95 & $\begin{array}{c}23,56 \\
- \\
26,35\end{array}$ & 55,62 & $\begin{array}{l}53,03 \\
- \\
58,21\end{array}$ \\
\hline $\begin{array}{c}\text { Junho a } \\
\text { Agosto/2016 }\end{array}$ & 6,67 & $\begin{array}{c}0,02 \\
- \\
13,32\end{array}$ & 23,67 & $\begin{array}{c}23,28 \\
- \\
24,07\end{array}$ & 57,55 & $\begin{array}{c}55,39 \\
- \\
59,72\end{array}$ \\
\hline
\end{tabular}


Tabela 3. Percentual de mortalidade de larvas do Aedes aegypti, em relação ao tempo de exposição às diferentes concentrações dos óleos essenciais obtidos da parte aérea de Croton tetradenius, coletadas nos meses de fevereiro, maio e agosto

\begin{tabular}{|c|c|c|c|c|c|c|c|c|c|c|c|}
\hline \multirow{3}{*}{\multicolumn{2}{|c|}{$\begin{array}{l}\text { Concentrações } \\
\left(m g \mathrm{~mL}^{-1}\right) / \text { Coleta }\end{array}$}} & \multicolumn{10}{|c|}{ Mortalidade (\%) } \\
\hline & & \multicolumn{2}{|c|}{$2 h$} & \multicolumn{2}{|c|}{$4 h$} & \multicolumn{2}{|c|}{$8 h$} & \multicolumn{2}{|c|}{$16 \mathrm{~h}$} & \multicolumn{2}{|c|}{$24 h$} \\
\hline & & $\begin{array}{l}\text { Mort. } \\
(\%)\end{array}$ & $I . C^{2}$ & $\begin{array}{l}\text { Mort. } \\
(\%)\end{array}$ & I.C. ${ }^{2}$ & $\begin{array}{l}\text { Mort. } \\
(\%)\end{array}$ & $I . C^{2}{ }^{2}$ & $\begin{array}{c}\text { Mort. } \\
(\%)\end{array}$ & $I . C^{2}$ & $\begin{array}{l}\text { Mort. } \\
(\%)\end{array}$ & I.C. ${ }^{2}$ \\
\hline \multirow{3}{*}{0,50} & Fevereiro & $0,00^{b}$ & ------ & $63,33^{a}$ & $\begin{array}{c}52,71 \\
- \\
72,95\end{array}$ & $97,50^{\mathrm{a}}$ & $\begin{array}{c}92,60 \\
- \\
102,39\end{array}$ & $100,00^{a}$ & ----- & $100,00^{a}$ & $\begin{array}{l}----- \\
\end{array}$ \\
\hline & Maio & $1,67^{\mathrm{a}}$ & $\begin{array}{c}0,22 \\
- \\
3,55\end{array}$ & $21,66^{\mathrm{a}}$ & $\begin{array}{c}6,96 \\
- \\
36,37\end{array}$ & $100,00^{a}$ & ------ & $100,00^{a}$ & ------ & $100,00^{a}$ & ------ \\
\hline & Agosto & $25,00^{a}$ & $\begin{array}{c}6,05 \\
- \\
43,95\end{array}$ & $100,00^{a}$ & ------ & $100,00^{a}$ & ------ & $100,00^{a}$ & ------ & $100,00^{\mathrm{a}}$ & ------ \\
\hline \multirow{3}{*}{0,25} & Fevereiro & $0,00^{b}$ & $\begin{array}{l}----- \\
\end{array}$ & $0,00^{\mathrm{b}}$ & $\begin{array}{l}----- \\
\end{array}$ & $22,49^{b}$ & $\begin{array}{c}10,17 \\
- \\
34,83\end{array}$ & $36,66^{b}$ & $\begin{array}{c}21,35 \\
- \\
51,99\end{array}$ & $46,66^{b}$ & $\begin{array}{c}31,34 \\
- \\
61,99\end{array}$ \\
\hline & Maio & $0,00^{b}$ & ----- & $0,00^{\mathrm{b}}$ & $\begin{array}{l}----- \\
\end{array}$ & $1,66^{b}$ & $\begin{array}{c}1,60 \\
- \\
4,93\end{array}$ & $5,83^{b}$ & $\begin{array}{c}2,70 \\
- \\
8,96\end{array}$ & $8,33^{b}$ & $\begin{array}{c}5,06 \\
- \\
11,59\end{array}$ \\
\hline & Agosto & $0,00^{b}$ & $\begin{array}{l}----- \\
\end{array}$ & $0,83^{\mathrm{b}}$ & $\begin{array}{c}0,39 \\
- \\
2,06\end{array}$ & $21,66^{b}$ & $\begin{array}{c}13,89 \\
- \\
29,44\end{array}$ & $55,83^{b}$ & $\begin{array}{c}44,09 \\
- \\
67,57\end{array}$ & $80,00^{b}$ & $\begin{array}{c}76,73 \\
- \\
83,27\end{array}$ \\
\hline \multirow{3}{*}{0,125} & Fevereiro & $0,00^{b}$ & ------ & $0,00^{b}$ & ------ & $0,00^{C}$ & ----- & $0,00^{C}$ & ----- & $0,00^{C}$ & ----- \\
\hline & Maio & $0,00^{b}$ & ----- & $0,00^{\mathrm{b}}$ & $\begin{array}{l}----- \\
\end{array}$ & $0,00^{6}$ & ----- & $0,00^{C}$ & ----- & $0,00^{C}$ & ----- \\
\hline & Agosto & $0,00^{b}$ & ----- & $0,00^{b}$ & ----- & $0,00^{C}$ & ------ & $0,00^{C}$ & ------ & $0,00^{C}$ & ----- \\
\hline \multirow{3}{*}{0,062} & Fevereiro & $0,00^{b}$ & ----- & $0,00^{b}$ & ----- & $0,00^{C}$ & $\begin{array}{l}---- \\
-1\end{array}$ & $0,00^{C}$ & ----- & $0,83^{\mathrm{C}}$ & $\begin{array}{c}0,79 \\
- \\
2,46\end{array}$ \\
\hline & Maio & $0,00^{b}$ & ------ & $0,00^{b}$ & ----- & $0,00^{b}$ & ----- & $0,00^{C}$ & ----- & $0,00^{C}$ & ------ \\
\hline & Agosto & $0,00^{b}$ & ------ & $0,00^{b}$ & ------ & $0,00^{C}$ & ------ & $0,00^{C}$ & ------ & $0,00^{C}$ & ----- \\
\hline
\end{tabular}

(Continua)

'Percentuais médios de mortalidade em relação às diferentes concentrações, seguidos pela mesma letra, nas colunas, dentro de um mesmo mês de coleta, não diferem significativamente pelo teste de Tukey, ao nível de $5 \%$ de probabilidade. ${ }^{2}$ I.C. $=$ Intervalo de confiança 
Tabela 3 (Continuação)

\begin{tabular}{|c|c|c|c|c|c|c|c|c|c|c|c|}
\hline \multirow{2}{*}{\multicolumn{2}{|c|}{$\begin{array}{c}\text { Concentrações } \\
\left(m g \mathrm{~mL}^{-1}\right) / \text { Coleta }\end{array}$}} & \multicolumn{10}{|c|}{ MORTALIDADE (\%) } \\
\hline & & \multicolumn{2}{|c|}{$2 h$} & \multicolumn{2}{|c|}{$4 h$} & \multicolumn{2}{|c|}{$8 h$} & \multicolumn{2}{|c|}{$16 h$} & \multicolumn{2}{|c|}{$24 h$} \\
\hline & & $\begin{array}{l}\text { Mort. } \\
\text { (\%) }\end{array}$ & $I . C .^{2}$ & $\begin{array}{l}\text { Mort. } \\
(\%)\end{array}$ & I.C. & $\begin{array}{l}\text { Mort. } \\
(\%)\end{array}$ & I.C. ${ }^{2}$ & $\begin{array}{l}\text { Mort. } \\
\text { (\%) }\end{array}$ & I..$^{2}$ & $\begin{array}{l}\text { Mort. } \\
(\%)\end{array}$ & $I . C^{2}$ \\
\hline \multirow{3}{*}{0,031} & Fevereiro & $0,00^{b}$ & ------ & $0,00^{b}$ & ----- & $0,00^{\mathrm{C}}$ & ------ & $0,00^{\mathrm{C}}$ & ----- & $0,00^{\mathrm{C}}$ & ----- \\
\hline & Maio & $0,00^{b}$ & ----- & $0,00^{b}$ & ----- & $0,00^{b}$ & ------ & $0,00^{c}$ & ----- & $0,00^{c}$ & ------ \\
\hline & Agosto & $0,00^{b}$ & ----- & $0,00^{b}$ & ----- & $0,00^{C}$ & ------ & $0,00^{C}$ & ----- & $0,00^{C}$ & ----- \\
\hline \multirow{3}{*}{0,019} & Fevereiro & $0,00^{b}$ & ----- & $0,00^{b}$ & ----- & $0,83^{c}$ & $\begin{array}{c}0,79 \\
- \\
2,46\end{array}$ & $0,83^{\mathrm{c}}$ & $\begin{array}{c}0,79 \\
- \\
2,46\end{array}$ & $0,83^{\mathrm{C}}$ & $\begin{array}{c}0,79 \\
- \\
2,46\end{array}$ \\
\hline & Maio & $0,00^{b}$ & ------ & $0,00^{b}$ & ----- & $0,00^{C}$ & ------ & $0,00^{c}$ & ----- & $0,00^{C}$ & ----- \\
\hline & Agosto & $0,00^{b}$ & ------ & $0,00^{b}$ & ----- & $0,00^{c}$ & ----- & $0,00^{c}$ & ----- & $0,00^{c}$ & $\begin{array}{ll}----- \\
\end{array}$ \\
\hline \multirow{3}{*}{0,007} & Fevereiro & $0,83^{b}$ & $\begin{array}{c}0,79 \\
- \\
2,46\end{array}$ & $0,83^{b}$ & $\begin{array}{c}0,79 \\
- \\
2,46\end{array}$ & $0,83^{\mathrm{c}}$ & $\begin{array}{c}0,79 \\
- \\
2,46\end{array}$ & $1,66^{\mathrm{c}}$ & $\begin{array}{c}0,22 \\
- \\
3,55\end{array}$ & $1,66^{\mathrm{c}}$ & $\begin{array}{c}0,22 \\
- \\
3,55\end{array}$ \\
\hline & Maio & $0,00^{b}$ & ----- & $0,00^{b}$ & ----- & $0,00^{b}$ & ----- & $0,00^{c}$ & ----- & $0,00^{c}$ & ----- \\
\hline & Agosto & $0,00^{b}$ & 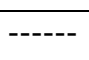 & $0,00^{b}$ & ----- & $0,00^{C}$ & ------ & $0,00^{C}$ & ----- & $0,00^{C}$ & ----- \\
\hline \multirow{4}{*}{0,003} & Fevereiro & $0,83^{b}$ & $\begin{array}{c}0,79 \\
- \\
2,46\end{array}$ & $0,83^{b}$ & $\begin{array}{c}0,79 \\
- \\
2,46\end{array}$ & $0,83^{c}$ & $\begin{array}{c}0,79 \\
- \\
2,46\end{array}$ & $1,66^{c}$ & $\begin{array}{c}0,22 \\
- \\
3,55\end{array}$ & $3,33^{c}$ & $\begin{array}{c}1,29 \\
- \\
7,95\end{array}$ \\
\hline & Maio & $0,00^{b}$ & ------ & $0,00^{b}$ & ------ & $0,00^{b}$ & ------ & $0,00^{c}$ & ------ & $0,00^{C}$ & ------ \\
\hline & Agosto & $0,00^{b}$ & ------ & $0,00^{b}$ & ----- & $0,00^{C}$ & ------ & $0,00^{C}$ & ----- & $0,00^{C}$ & ----- \\
\hline & Fevereiro & 0,00 & ------ & $0,00^{b}$ & ----- & $0,00^{c}$ & ------ & $0,00^{c}$ & ------ & $0,00^{c}$ & ----- \\
\hline \multirow{2}{*}{$C T L$} & Maio & $0,00^{b}$ & ----- & $0,00^{b}$ & ------ & $0,00^{b}$ & ------ & $0,00^{c}$ & ----- & $0,00^{c}$ & ------ \\
\hline & Agosto & $0,00^{b}$ & ------ & $0,00^{b}$ & ------ & $0,00^{c}$ & ------ & $0,00^{c}$ & ------ & $0,00^{c}$ & ----- \\
\hline
\end{tabular}

${ }^{1}$ Percentuais médios de mortalidade seguidos pela mesma letra nas colunas, dentro de um mesmo mês de coleta, não diferem significativamente pelo teste de Tukey, ao nível de 5\% de probabilidade. ${ }^{2}$ I.C. $=$ Intervalo de confiança 
Tabela 4. Classificação dos compostos químicos encontrados nos óleos essenciais obtidos da parte aérea de Croton tetradenius, cujas coletas foram realizadas nos meses de Fevereiro, Maio e Agosto, de 2016, na Floresta Nacional Contendas do Sincorá, Bahia, Brasil

\begin{tabular}{lccc}
\hline \multirow{2}{*}{ Constituinte } & \multicolumn{3}{c}{ C. tetradenius (\%) } \\
\cline { 2 - 4 } & Fevereiro & Maio & Agosto \\
\hline Compostos encontrados & 60 & 48 & 62 \\
Compostos identificados (\%) & 61,66 & 62,50 & 58,06 \\
Monoterpenos hidrocarbonados (\%) & 37,83 & 40,00 & 38,88 \\
Monoterpenos oxigenados (\%) & 35,13 & 26,66 & 36,11 \\
Sesquiterpenos hidrocarbonados (\%) & 16,21 & 20,00 & 16,66 \\
Sesquiterpenos oxigenados (\%) & 10,81 & 13,33 & 8,33 \\
\hline
\end{tabular}


Tabela 5. Composição química dos óleos essenciais obtidos da parte aérea de Croton tetradenius, realizada através do método de cromatografia gasosa acoplada à espectrometria de massas, cujas coletas foram realizadas nos meses de Fevereiro, Maio e Agosto de 2016, na Floresta Nacional Contendas do Sincorá, Bahia, Brasil

\begin{tabular}{|c|c|c|c|c|c|}
\hline \multirow{2}{*}{ Constituinte } & \multirow{2}{*}{$I R L^{1}$} & \multirow{2}{*}{$I K^{2}$} & \multicolumn{3}{|c|}{ C. tetradenius $(\%)^{3}$} \\
\hline & & & Fevereiro & Maio & Agosto \\
\hline Tricicleno & $929-930$ & 926 & 1,25 & 0,23 & 1,53 \\
\hline$\alpha$-Tujeno & $930-931$ & 930 & 1,25 & --- & 0,60 \\
\hline a-Pineno & 940 & 939 & 3,21 & 0,94 & 3,88 \\
\hline Canfeno & 057-958 & 954 & 2,72 & 0,92 & 3,50 \\
\hline Sabineno & $978-979$ & 975 & 1,29 & 0,39 & 0,75 \\
\hline$\beta$-Pineno & $983-984$ & 979 & 0,83 & 0,21 & 0,76 \\
\hline Mirceno & $992-993$ & 990 & 4,38 & 1,80 & 3,05 \\
\hline$\alpha$-Felandreno & $1011-1012$ & 1002 & 3,05 & 0,79 & 1,37 \\
\hline$\alpha$-Terpineno & $1022-1025$ & 1017 & 6,99 & 1,78 & 2,24 \\
\hline p-Cimeno & 1033-1034 & 1024 & 5,52 & 6,95 & 7,85 \\
\hline Limoneno & $1036-1038$ & 1029 & 3,45 & 1,96 & 3,51 \\
\hline 1,8-Cineol & $1038-1039$ & 1031 & 0,05 & --.- & 0,08 \\
\hline (E)- $\beta$-Ocimeno & 1051-1952 & 1050 & 0,88 & 0,27 & 0,23 \\
\hline y-Terpineno & $1065-1066$ & 1059 & 4,72 & 3,44 & 3,15 \\
\hline$\sigma-2-C a r e n o$ & $1089-1090$ & 1002 & 1,11 & ---- & 0,27 \\
\hline trans-p-Menta-2,8-dien-1-ol & $1132-1133$ & 1122 & 0,87 & ---- & 0,65 \\
\hline cis-p-Menta -2,8-dien-1-ol & 1150 & 1137 & 0,16 & ---- & 0,21 \\
\hline Cânfora & $1162-1164$ & 1146 & 18,18 & 30,95 & 23,91 \\
\hline Isoborneol & $1173-1174$ & 1160 & 2,34 & 4,54 & 2,34 \\
\hline Pinocarvone & $1177-1178$ & 1164 & 0,43 & ---- & 0,46 \\
\hline Borneol & $1180-1182$ & 1169 & 0,63 & 0,78 & 0,35 \\
\hline Terpineno-4-ol & $1187-1189$ & 1177 & 2,57 & 3,66 & 2,31 \\
\hline p-Cimeno-8-ol & 1194 & 1182 & 0,66 & --- & -- \\
\hline$\alpha$-Terpineol & 1201 & 1188 & 0,46 & ---- & 0,56 \\
\hline $\mathrm{y}$-Terpineol & $1253-1254$ & 1199 & 9,76 & 12,70 & 16,00 \\
\hline Carvacrol & $1298-1308$ & 1299 & --- & 0,45 & 0,58 \\
\hline Mirtenil acetato & $1328-1330$ & 1326 & 0,60 & 1,01 & 0,50 \\
\hline$\alpha$-Terpinil acetato & $1352-1354$ & 1349 & 2,15 & 3,07 & 1,48 \\
\hline a-Copaeno & $1381-1382$ & 1376 & 2,04 & 2,98 & 1,78 \\
\hline$\beta$-Cariofileno & $1425-1427$ & 1419 & 1,05 & 1,16 & 0,63 \\
\hline (Z)- $\beta$-Farneseno & $1462-1467$ & 1442 & 0,73 & 0,74 & 0,34 \\
\hline$\alpha$-Humuleno & $1455-1463$ & 1454 & 1,66 & 1,74 & 1,04 \\
\hline Biciclogermacreno & $1500-1502$ & 1500 & 1,32 & 0,67 & 0,32 \\
\hline$\sigma$-Cadineno & 1523-1525 & 1523 & 0,33 & 0,19 & 0,13 \\
\hline (E)-Nerolidol & $1567-1581$ & 1563 & 0,13 & 0,19 & --- \\
\hline Espatulenol & $1585-1587$ & 1578 & 1,17 & 0,83 & 0,66 \\
\hline$\beta$-Copaen-4- $\alpha$-ol & $1596-1598$ & 1590 & 0,56 & 0,62 & 0,42 \\
\hline Epi- $\alpha$-cadinol & $1650-1653$ & 1640 & 0,55 & 0,23 & 0,23 \\
\hline Não identificados (\%) & & & $0,11-1,34$ & $0,21-3,02$ & $0,07-2,12$ \\
\hline
\end{tabular}

Tíndices de retenção com coluna capilar Factor Four/VF-5ms. ${ }^{2}$ Indices de Kovats em coluna capilar DB-5 (ADAMS, 2007). ${ }^{3}$ Substâncias listadas por ordem de eluição em coluna capilar Factor Four/VF-5ms. 